\title{
Jets and perfect liquid at LHC and RHIC
}

\author{
Julia Velkovska*t \\ Department of Physics and Astronomy, Vanderbilt University, Nashville, TN 37235, USA \\ E-mail: julia.velkovska@vanderbilt.edu
}

The quark-gluon plasma is a state of matter comprised of deconfined quarks and gluons that exhibits remarkable properties. It has vanishingly small specific viscosity making it one of the most perfect liquids known. It also has a record-high density, and is extremely opaque to transiting particles. Experimentally, the perfect liquid manifests itself in a collective flow of the produced particles that retains memory of the geometry of the initial-state energy density distribution, while the opacity is observed through the quenching of jets. This talk presents an overview of some of the historic hard-probe measurements at RHIC and the more recent results from the energy frontier with the LHC. It then discusses collective flow measurements from both RHIC and LHC, where we explore the limits of the perfect liquid behavior in the smallest quark-gluon plasma droplets.

The 4th KMI International Symposium (KMI2019)

18-20, February 2019

Nagoya, Japan

\footnotetext{
* Speaker.

${ }^{\dagger}$ I thank the Kobayashi-Maskawa Institute for the local support and my host, Prof. Chiho Nonaka, for the invitation and warm hospitality. This work was supported in part by the US Department of Energy grant DE-FG02-92ER40712.
} 


\section{Introduction}

The study of the quark-gluon plasma (QGP), a state of matter comprised of deconfined quarks and gluons, is the main objective of the relativistic heavy ion programs at the Relativistic Heavy Ion Collider (RHIC) at Brookhaven National Laboratory in the USA, and at the Large Hadron Collider (LHC) at CERN (Switzerland). After nearly two decades of experiments, these programs have established that the QGP has remarkable properties, such as record-high density making it extremely opaque to quarks and gluons, and very low shear viscosity over entropy density $\eta / s$. This is a brief review of the status of our understanding of how these properties emerge and evolve with system size and various other experimentally accessible quantities.

\section{Jet quenching in QGP}

Jets are produced in hard-scattering processes with large momentum transfer between the participating partons. Due to the hard scales involved, they are formed in the early stage of the collisions and the outgoing quarks and gluons traverse the medium formed in heavy ion collisions and sense its properties. They may suffer energy loss through induced gluon radiation or through elastic collisions. The production rate can be calculated within perturbative QCD, and the production in proton-proton collisions can serve as a reference since QGP is not expected to be formed in such collisions. Jet quenching was first discovered at RHIC through the observation of suppression of production of high-momentum hadrons in $\mathrm{Au}+\mathrm{Au}$ collisions [1] quantified by the nuclear modification factor $R_{\mathrm{AA}}$. Over the years, a wealth of measurements with ever-increasing precision have been published for both inclusive and identified hadrons produced in nucleus-nucleus (AA or $\mathrm{AB}$ ) collisions at RHIC and LHC. Figure 1 presents a compilation of $R_{\mathrm{AA}}$ results for hadrons and for electroweak bosons. As expected, photons, $\mathrm{Z}$ and $\mathrm{W}$ bosons are not suppressed, since they do not interact strongly. Remarkably, heavy-flavor hadrons are also strongly suppressed, which was initially unexpected. A number of theoretical developments were undertaken to improve the description of the heavy flavor hadron and jet suppression [2]. Presently, the precise measurement of charm and beauty energy loss is a major goal of the experimental programs both at RHIC and at the LHC.

Further important control experiments that contributed to the jet-quenching interpretation of the observed hadron suppression are the measurements of dihadron correlations and nuclear modification factors in $\mathrm{d}+\mathrm{Au}$ and in $\mathrm{p}+\mathrm{A}$ collisions, such as those shown in Fig. 2. Away-side hadrons (e.g. - those that traverse a longer path length through the medium) are suppressed in $\mathrm{Au}+\mathrm{Au}$, but not in $\mathrm{d}+\mathrm{Au}$ collisions. Similarly, the hadron nuclear modification factors are compatible with unity in $\mathrm{d}+\mathrm{Au}$ and $\mathrm{p}+\mathrm{Pb}$ collisions, as predicted for a cold nuclear medium where QGP is not formed. As discussed in Sec. 3 until recently it has been the prevailing paradigm that small-system collisions can not form QGP due to the short lifetime and small volume of the system. However, this paradigm is now under critical assessment due to multiple measurements from the soft sector indicating collective effects and near-perfect fluid behavior. In terms of jet quenching, the null effect in $\mathrm{d}+\mathrm{Au}$ and $\mathrm{p}+\mathrm{A}$ may just mean that the parton path length through the medium formed in these system is not large enough for sizable jet-quenching to occur. 

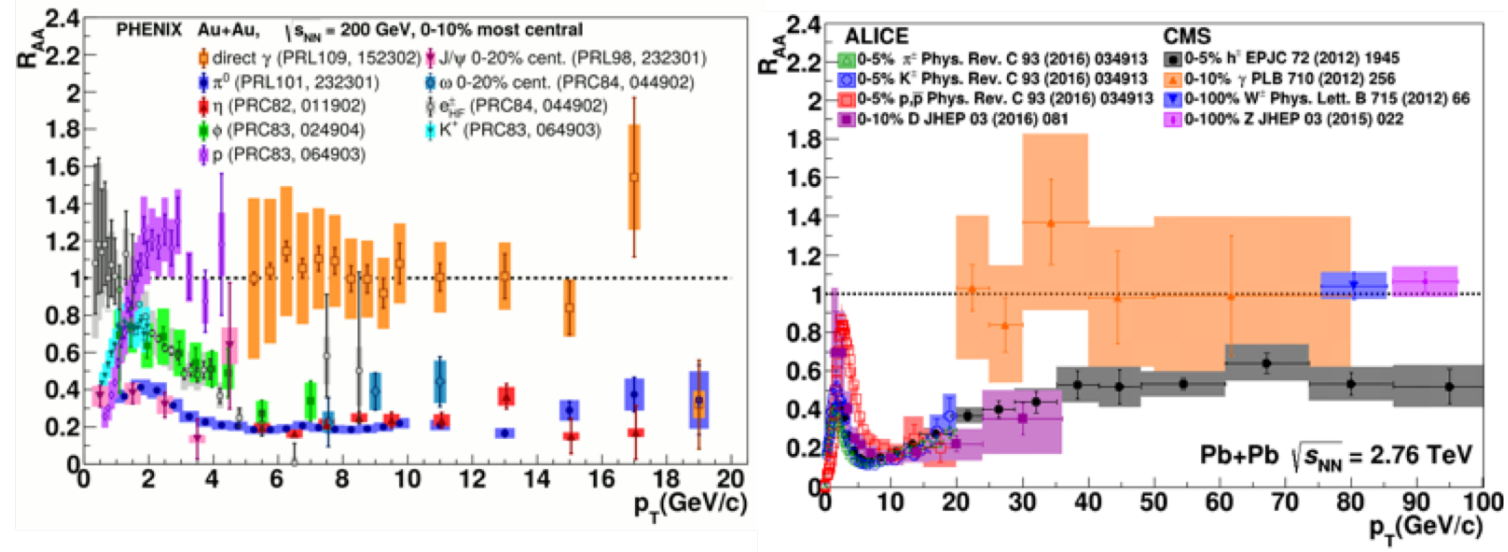

Figure 1: Nuclear modification factors hadrons, and electroweak bosons measured at RHIC (left) and at the LHC (right). References to the original publications are given in the legend.
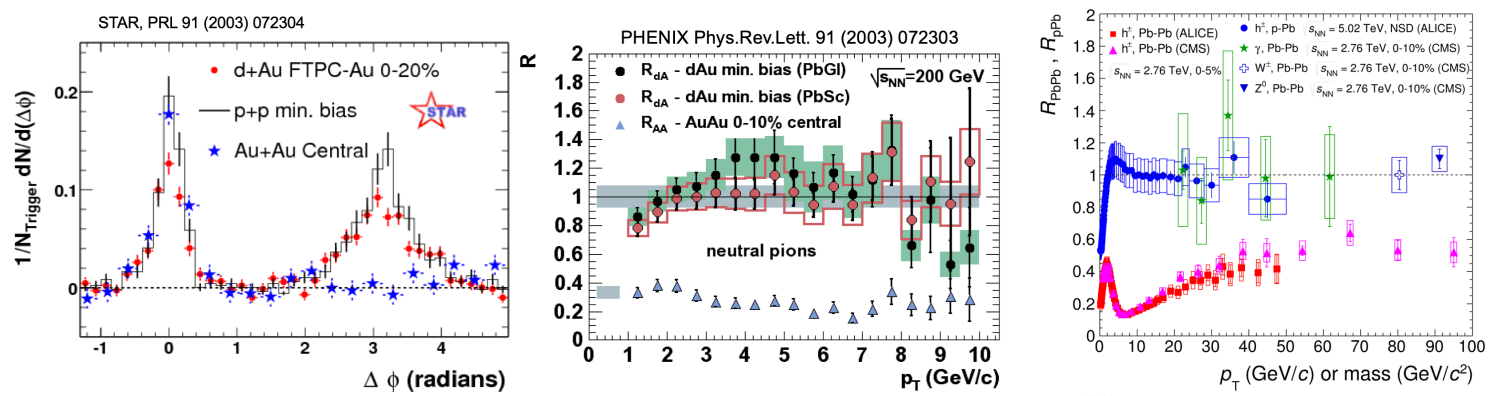

Figure 2: Dihadron correlations measured in $p+p d+A u$ and in $A u+A u$ collisions [5] (left panel). Comparison between hadron nuclear modification factors measured in $\mathrm{d}+\mathrm{Au}$, and $\mathrm{Au}+\mathrm{Au}$ collisions at RHIC [6] (middle panel), as well as $\mathrm{p}+\mathrm{Pb}$ and $\mathrm{Pb}+\mathrm{Pb}$ collisions at the LHC [7] (right panel).

The $R_{\mathrm{AA}}$ measurements have provided a fertile ground for developments in the jet-quenching phenomenology, which over the years has evolved to include realistic hydrodynamically expanding medium, dynamical jet-medium interactions, multi-scale running coupling effects for radiative and elastic energy loss, including fluctuations. The $R_{\mathrm{AA}}$ data have been used [2] to quantify the QGP properties by extracting the jet-quenching transport coefficient $\hat{q}$, which specifies the averaged transverse momentum squared transferred between the jet and the medium per unit length.

The LHC, with its high center-of-mass energy and sophisticated large-coverage jet detection brought exiting new dimensions to jet-quenching measurements in QGP. For the first time, jetquenching was directly observed in dijet imbalance measurements $[3,4]$ and a detailed study was performed to investigate and account for the lost energy [4]. One disadvantage of using dijet events to study parton energy loss is that both partons are traversing the medium. Thus, only the difference in energy loss can be measured. Correlation measurements between electroweak bosons that do not lose energy in the medium and jets give a better handle on the lost energy, albeit with a much smaller statistical precision due to the small production rate. Examples of such measurements $[8,9,10]$ are shown in Fig. 3. A clear shift in the mean of the distribution of the jet-boson transverse momentum ratio is observed comparing the measurements in $\mathrm{pp}$ and $\mathrm{PbPb}$ collisions, from which the lost 
energy can be assessed.
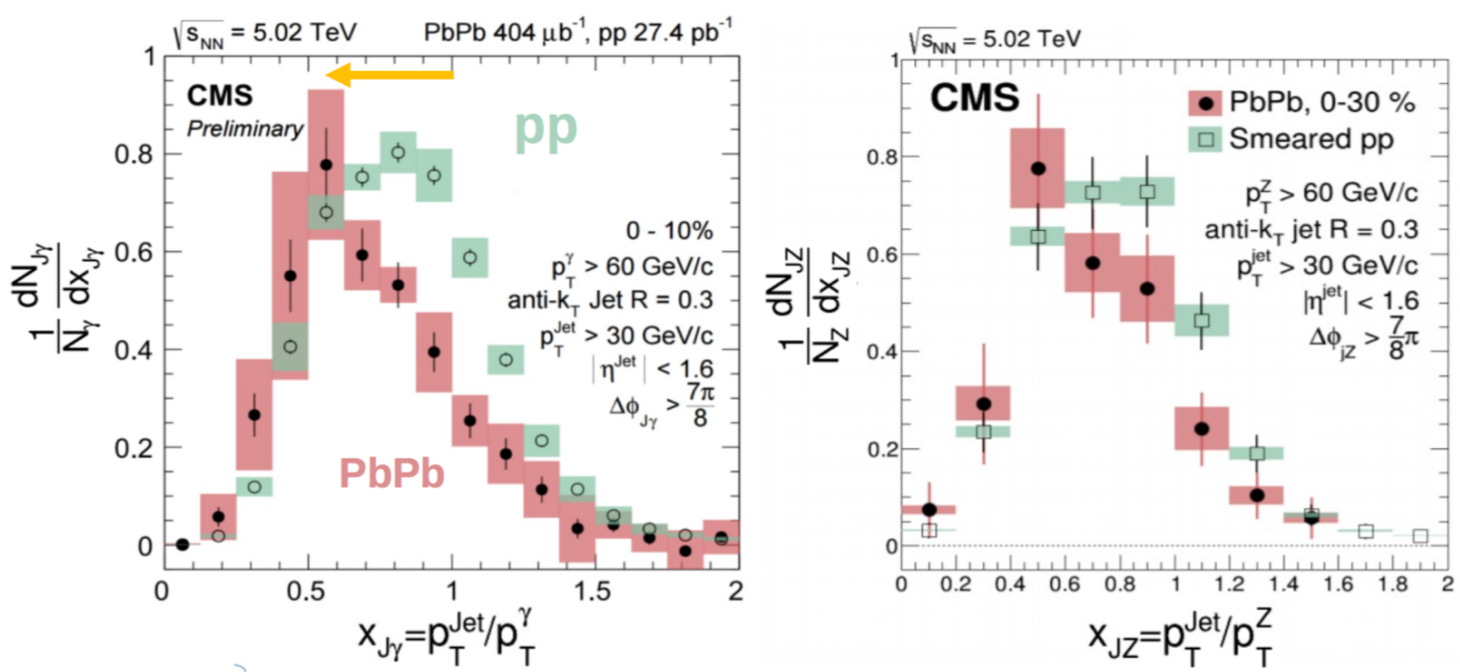

Figure 3: Distributions of the transverse momentum ratio $x_{j \gamma}$ and $x_{j Z}$ between the jet and the isolated photon [8], or the $\mathrm{Z}$ boson [10].

The detailed understanding of jet-medium interactions is a complex problem that involves both perturbative and non-perturbative physics. The energy loss process, the redistribution and possible thermalization of the lost energy, the medium response, and how the jet observables are influenced by the soft underlying event background that fluctuates on an event-by-event basis, are under intense theoretical study. In recent years, the LHC experiments have delivered a number of jet structure measurements in $\mathrm{pp}$ and $\mathrm{PbPb}$ collisions and evaluated the modifications induced by the QGP medium. These include the measurements of the jet fragmentation functions [11], jet shape [12], jet mass [13], and the groomed jet splitting functions [14]. A consistent theoretical description of all measurements is still under development [15]. At the time of this writing, the JETSCAPE collaboration has released a comprehensive computational envelope for developing complete event generators for heavy-ion collisions [16]. It package allows for modular incorporation of a wide variety of existing and future software modules that simulate different aspects of a heavy-ion collision. The goal of this project is to standardize the various physics elements required to simulate an entire event in a heavy-ion collisions, and to provide the tools for statistical comparison between data and theory. The present implementation includes various formalisms of parton energy loss that are based on different phase space assumptions, and therefore are becoming active at different parts of phase space in the jet evolution. This comprehensive approach requires tuning of multiparameter models through simultaneous comparisons with multiple experimental observables. This can only be achieved with modern computation and machine-learning techniques, which are now being developed to describe both the bulk medium [17] and jet-medium interactions [18].

It has been more than a decade since it was recognized that the QGP is not a weakly coupled parton gas but a strongly coupled, near-perfect liquid with a very low ratio of (shear) viscosity to entropy density $\eta / s$. At the same time, the liquid is extremely opaque to hard-scattered partons traversing through it. The jet-quenching transport coefficient $\hat{q}$ is closely related to $\eta / s$, as detailed in Ref. [19]: a large value of $\hat{q}$ implies a small $\eta / s$, indicative of strong coupling. Establishing 
this relation both experimentally and theoretically through studies of QGP over a wide range of length scales remains a major programmatic goal of the heavy ion community. To achieve this goal a new state-of-the-art jet detector called sPHENIX is under construction at RHIC [20] and a rich experimental program is being planned with the high-luminosity LHC and upgraded detectors at CERN [21].

\section{Liquid properties of QGP}

\subsection{Large systems}

After the initial success of ideal hydrodynamics in describing RHIC measurements of azimuthal anisotropies in hadron production quantified by the measurement of the second Fourier harmonic in the particle azimuthal distributions, $v_{2}$ (elliptic flow), the perfect fluid paradigm was adopted for the QGP. Vigorous work ensued to quantify precisely how perfect the liquid is and how this property emerges. Two major breakthroughs are worth noting: 1) development of viscous hydrodynamics [22], and 2) the realization [23] that even in symmetric nuclear collisions, the initial geometric distribution of the energy density is not smooth but fluctuates on an event-by-event basis, which then gives the possibility of measuring odd harmonics in the final-state particle distributions and probing different length scales in QGP. The subsequent measurements [24] of high-order harmonics, $v_{n}$ and the comparisons with event-by-event hydrodynamic calculations [25] brought a new level of precision in determining $\eta / s$. Shown in Fig. 4 is a comparison between $v_{n}\left(p_{T}\right)$ data from RHIC and LHC, and event-by-event viscous hydrodynamics calculations, which led to the conclusion that the QGP created at RHIC and LHC has $\eta / s$ of the order $1.2-0.2$, which is within a factor of $1.5-2.5$ of the minimum established in string theory.

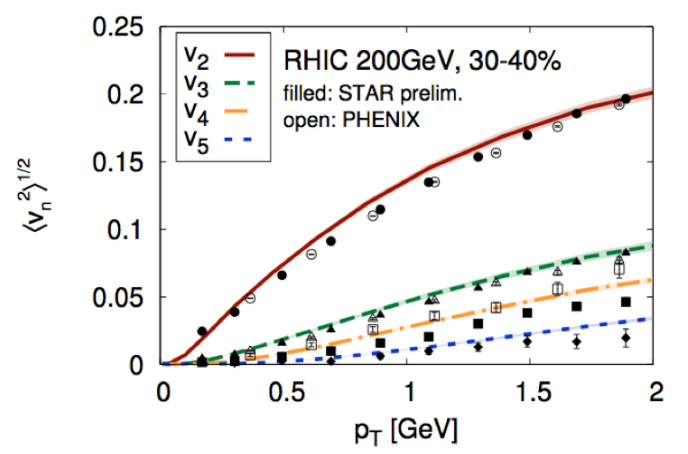

$$
\eta / s \approx 0.12 \text { at } \sqrt{s}=0.2 \mathrm{TeV}
$$

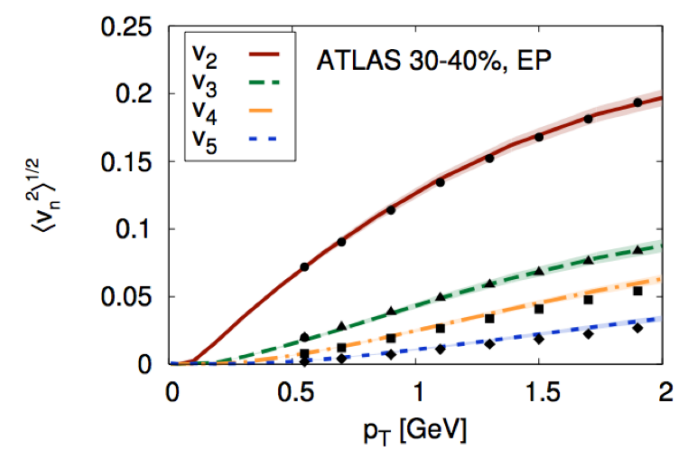

$\eta / s \approx 0.2$ at $\sqrt{s}=2.76 \mathrm{TeV}$

Figure 4: Measurements [24] of high-order flow harmonics at RHIC and LHC in comparison with hydrodynamic calculations [25].

\subsection{Small systems}

Recent observations of collective behavior in small collision systems at LHC [26, 27] and RHIC $[28,29,30]$ have challenged our understanding of the minimal conditions necessary for 
QGP formation, the importance of thermal equilibration and preequilibrium dynamics, and the limits of applicability of hydrodynamics. The initial observation of the so-called ridge structure in the two particle correlations in relative pseudorapidity $(\Delta \eta)$ and azimuthal angle separation $(\Delta \phi)$ led to a proliferation of theoretical models proposing non-QGP mechanisms to explain these longrange correlations. Fig. 5 shows examples of the observed two-particle correlations with the ridge structure indicated with the arrows.
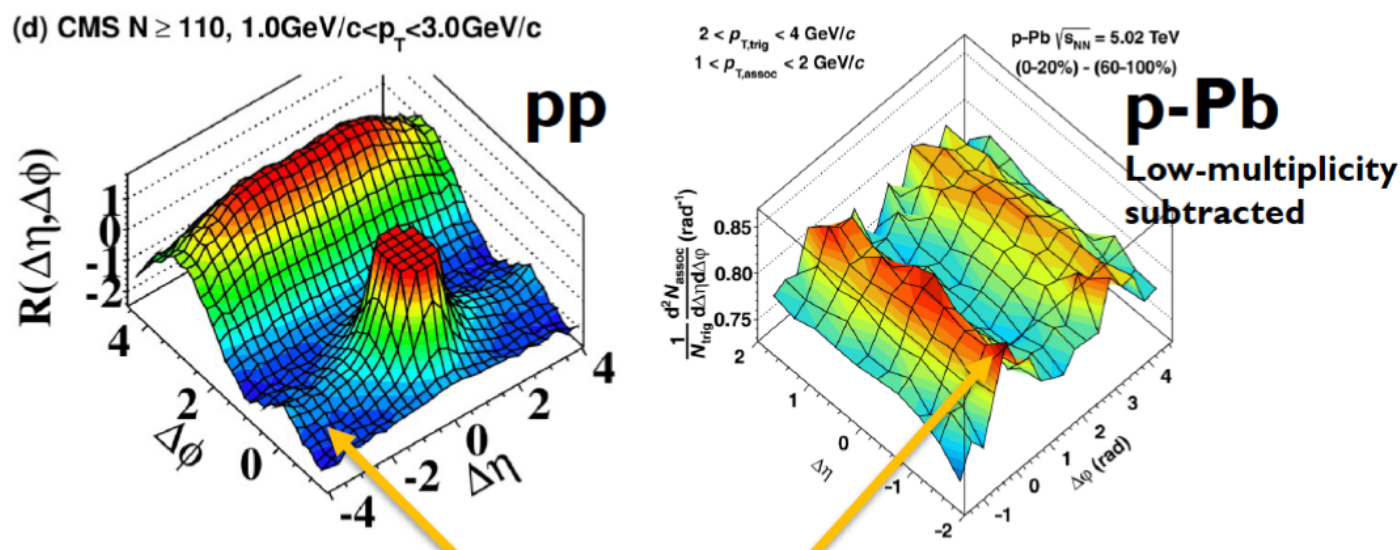

\section{CMS, JHEP 09 (2010) 091}

\section{Near side ridge}

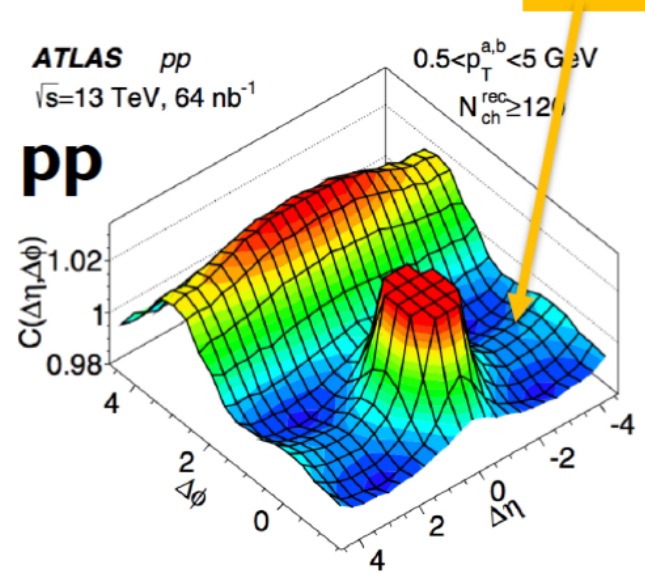

ATLAS, PRC 96, (2017) 024908

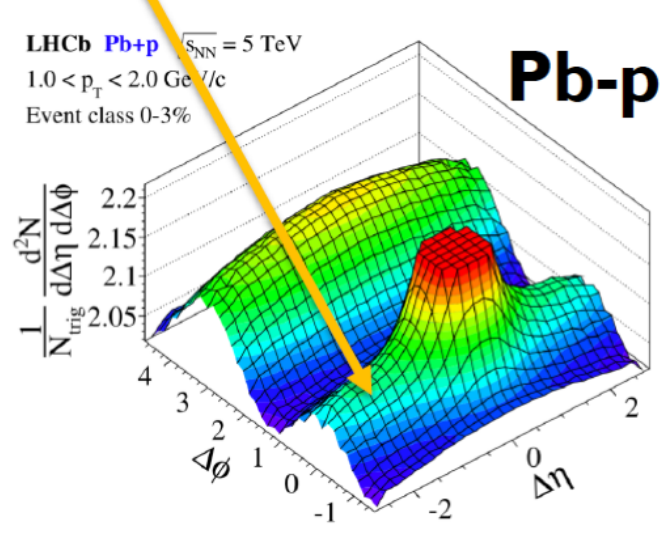

LHCb, PLB 762 (20I6) 473-483

Figure 5: LHC observations of a ridge structure in the two-particle $\Delta \eta-\Delta \phi$ correlations in small-system collisions.

It has been established that in large-ion collision the ridge in the two-particle correlations is a consequence of collective hydrodynamic flow in QGP. Observation of a ridge in small systems is suggestive that the same mechanism may be at play. However, keeping in mind that there are many processes that can induce correlations between just a few particles in the system, it was imperative to confirm that a common flow pattern is seen for all particles in the system. Measurements of the elliptic flow using multi-particle correlations [31] and the observation of a mass-dependent elliptic flow [32] confirmed the collective nature of the observed correlations. Does collectivity imply QGP formation? One way of testing the QGP hypothesis is to make use of its near per- 
fect fluid properties. If a small droplet of QGP is formed, it tends to preserve a memory of any initial-state geometric asymmetry in the energy density distribution and translates it to the finalstate particle momentum distribution. One can, therefore, engineer the shape of the final-state particle distributions by selecting projectile-target combinations with different initial geometry as proposed in Ref. [33]. In 2014-2016 the PHENIX experiment at RHIC performed a small-system geometry scan using $\mathrm{p}+\mathrm{Au}, \mathrm{d}+\mathrm{Au}$, and ${ }^{3} \mathrm{He}+\mathrm{Au}$ collisions at $\sqrt{s_{N N}}=200 \mathrm{GeV}$. The elliptic and triangular flow components, $v_{2}$ and $v_{3}$, were studied in the three systems. The $v_{2}\left(p_{T}\right)$ and $v_{3}\left(p_{T}\right)$ measurements are shown in Fig. 6, along with the initial-state elliptic and triangular eccentricities calculated using the Monte-Carlo Glauber model. As expected from the initial geometry, the $v_{2}^{\mathrm{p}+\mathrm{Au}}<v_{2}^{\mathrm{d}+\mathrm{Au}} \approx v_{2}^{3} \mathrm{He}+\mathrm{Au}$, and $v_{3}^{\mathrm{p}+\mathrm{Au}} \approx v_{3}^{\mathrm{d}+\mathrm{Au}}<v_{3}^{3} \mathrm{He}+\mathrm{Au}$. With this unique combination of initial geometries, mostly circular, elliptic, or triangular liquid droplets can be produced and then imprinted in the final-state particle distributions. In Fig. 7 the measurements are compared to two sets of hydrodynamics predictions [35, 36]. Both calculations include short-lived QGP droplets with $\eta / s=1 / 4 \pi$, the ADS/CFT limit. They provide a simultaneous description of two flow patterns in three different systems, with p-values that provide strong support for the QGP hypothesis [34]. Alternative theoretical descriptions that are based on initial-state momentum domain correlations have been ruled out [34, 37].

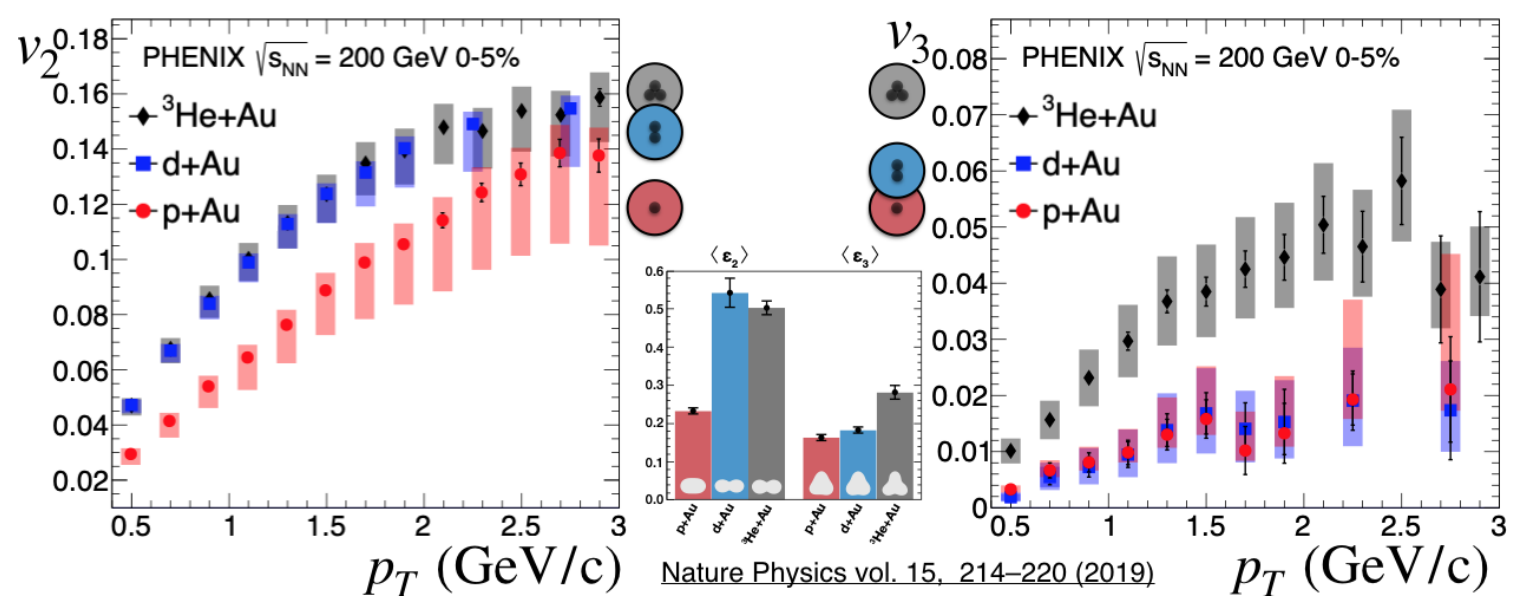

Figure 6: Elliptic (left) and triangular flow (right) measured in $0-5 \%$ central $\mathrm{p}+\mathrm{Au} \mathrm{d}+\mathrm{Au}$ and ${ }^{3} \mathrm{He}+\mathrm{Au}$ collisions at $\sqrt{s_{N N}}=200 \mathrm{GeV}$ by the PHENIX experiment [34] compared to the initial-state elliptic and triangular eccentricities calculated using MC Glauber model (middle panel).

\section{Summary}

This talk reviewed the studies of QGP at RHIC and LHC using jets and collective flow observed in different collision systems. Jet quenching and various modifications in the jet structure are observed in large-ion collisions at both facilities. Detailed experimental and theoretical studies are underway aiming to extract information about the underlying QGP properties and the physics processes governing its evolution. Amazingly, the nearly perfect liquid behavior appears to be ubiquitous from small to large systems, and viscous hydrodynamics with specific viscosity near 


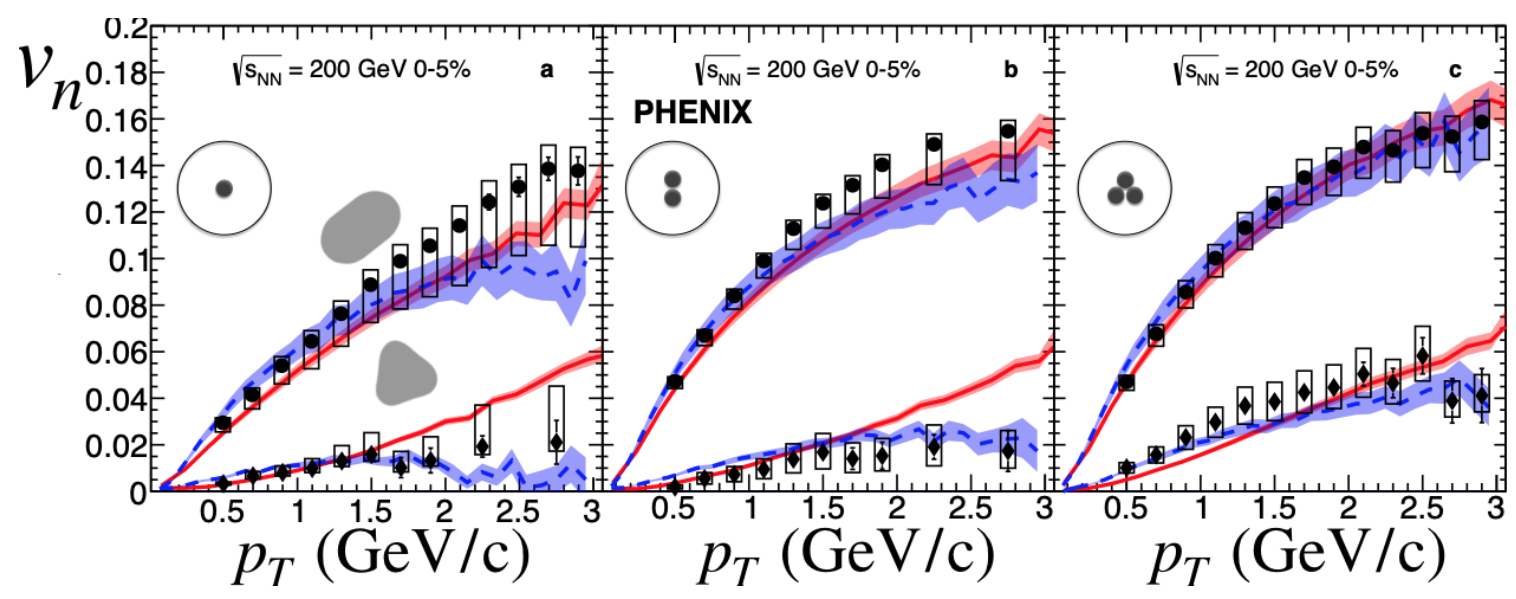

Figure 7: Elliptic (upper curves) and triangular flow (lower curves) measured in 0-5\% central p+Au, d+Au , and ${ }^{3} \mathrm{He}+\mathrm{Au}$ collisions at $\sqrt{s_{N N}}=200 \mathrm{GeV}$ by the PHENIX experiment [34] compared to predictions from hydrodynamic calculations $[35,36]$.

the ADS/CFT limit provides a simultaneous and quantitative description of a large body of data, despite the fact that thermal equilibrium is likely not achieved in the short-lived systems.

\section{References}

[1] PHENIX Collaboration, Phys. Rev. Lett. 88 (2002) 022301

[2] The JET Collaboration, Phys. Rev. C 90 (2014) 014909

[3] ATLAS Collaboration, Phys. Rev. Lett. 105 (2010) 252303

[4] CMS Collaboration, Phys. Rev. C 84 (2011) 024906

[5] STAR Collaboration, Phys. Rev. Lett. 91 (2003) 072304

[6] PHENIX Collaboration, Phys. Rev. Lett. 91 (2003) 072303

[7] N. Brambilla et al. Eur. Phys. J. C (2014) 74:2981

[8] CMS Collaboration, Phys. Lett. B 785 (2018) 14

[9] ATLAS Collaboration, Phys. Lett. B 789 (2019) 167

[10] CMS Collaboration, Phys. Rev. Lett. 119 (2017) 082301

[11] ATLAS Collaboration, Phys. Rev. C 98 (2018) 024908

[12] CMS Collaboration, Phys. Lett. B 730 (2014) 243

[13] ALICE Collaboration, Phys. Lett. B 776 (2018) 249

[14] CMS Collaboration, Phys. Rev. Lett. 120 (2018) 142302

[15] R. Elayavalli and K. Zapp, JHEP 1707 (2017) 141

[16] J.H. Putschke et al., arXiv:1903.07706

[17] Jonah E. Bernhard,et al., Phys. Rev. C 94 (2016) 024907

[18] Yayun He et al., Phys. Rev. Lett. 122 (2019) 252302 
[19] Abhijit Majumder et al., Phys. Rev. Lett. 99 (2007) 192301

[20] PHENIX Collaboration, arXiv:1501.06197

[21] Z. Citron et al., arXiv: 1812.06772

[22] Luzum, Romatschke, Phys. Rev. C 78 (2008) 034915

[23] B. Alver, G. Roland, Phys. Rev. C 81 (2010) 054905; Erratum-ibid. C82 (2010) 039903

[24] PHENIX Collaboration, Phys. Rev. Lett. 107 (2011) 252301; STAR Collaboration, Phys. Rev. C 88 (2013) 14904 ; ATLAS Collaboration, Phys. Rev. C 86 (2012) 014907; CMS Collaboration, Phys. Rev. C 89 (2014) 044906

[25] C. Gale et al., Phys. Rev. Lett. 110 (2013) 012302

[26] CMS Collaboration, PLB 718 (2013) 795-814

[27] CMS Collaboration, JHEP 09 (2019) 091; ATLAS Collaboration, Phys. Rev. Lett. 116 (2016) 172301

[28] PHENIX Collaboration, Phys. Rev. C 95 (2017) 034910

[29] PHENIX Collaboration, Phys. Rev. Lett. 114 (2015) 192301

[30] PHENIX Collaboration, Phys. Rev. Lett. 115 (2015) 142301

[31] CMS Collaboration, Phys. Lett. B 724 (2013) 213 - 240; Phys. Rev. Lett. 115 (2015) 012301

[32] ALICE Collaboration, Phys. Lett. B 726, 164 - 177 (2013); PHENIX Collaboration Phys. Rev. C 97 (2018) 064904

[33] J. L. Nagle et al., Phys. Rev. Lett. 113 (2014) 112301

[34] PHENIX Collaboration, Nature Phys. 15 (2019) 214

[35] P. Romatschke, Eur. Phys. J. C75 (2015) 305

[36] Chun Shen et al., Phys. Rev. C 95 (2017) 014906

[37] M. Mace et al.,Erratum:[Phys. Rev. Lett. 121, 052301(2018)], Phys. Rev. Lett. 123 (2019) 039901 